\title{
Integrating Cognitive and Collective Aspects of Work in Evaluating Technology
}

\author{
Anne-Sophie Nyssen
}

\begin{abstract}
Ergonomic criteria are receiving increasing attention from designers but their applications do not ensure that technology matches the user's abilities, needs, and work constraints. In this paper, we used two series of studies conducted in the healthcare system to illustrate how important work analysis is in evaluating new technology in order to identify critical dimensions of work and anticipate the impact of the devices on these dimensions: one looking at a new automatic drug device for anesthetists and one at a new robotic surgery system. Given the results, we developed a methodology for evaluation of new technology in complex systems. The aim is to insist to assess the impacts of technology on all the dimensions of work: technical, performance, cognitive, organizational, and economic. Which dimensions and criteria will be developed more in detail depends on the results of the work analysis.
\end{abstract}

Index Terms-Cognitive and collective work, evaluating health technology, naturalistic study, robotic.

\section{INTRODUCTION}

$\mathbf{T}$ HE NUMBER, complexity, and variety of medical devices have increased in recent years. At the same time, human error is considered to be the major contributing factor of medical accidents. Accident investigations are traditionally based on epidemiological methods rather than on detailed analyses of work situations. These methods often classify accidents into exclusive categories: human error, equipment failure, or unavoidable complication. We can ask ourselves if such a classification still makes sense in our modern world where human techniques and organization are interdependent. The healthcare system is characterized by diversity, complexity, and the need for coordinated work between multiple disciplines. This has caused great difficulty in the design of clinical technical systems. Designers can be some kind of dreamers; they discover how difficult it is to assist activity in naturalistic situations. Many technical aids are not used, are misused, or induce new forms of errors. This paradox was depicted by Bainbridge [1] for automated systems as the irony of automation. Among the reasons for these failures we can quote [2]:

1) a large mismatch between aid support and users' real needs;

2) the communication gap between potential users and computer science, where the role of the aid is often unclear for the user;

Manuscript received April 16, 2004; revised July 19, 2004. This work was supported in part by the FNRS and in part by the Belgian Federal Government, Prime Minister's Offices, OSTC Program Phase II (Worker protection in the area of health). This paper was recommended by the guest editors of this special issue.

The author is with the Department of Work Psychology, University of Liège, 4000 Liège, Belgium (e-mail: asnyssen@ulg.ac.be).

Digital Object Identifier 10.1109/TSMCA.2004.836800
3) the absence of a coherent design philosophy: for instance, the method of knowledge representation may be inappropriate;

4) the disregard of organizational issues: the complex environment where the system is used is not taken into account, nor are its dynamics and uncertainty.

Regarding the unintended side effects of technology, several researchers have indicated the need to reevaluate the human-machine interaction at a fundamental level [3]-[6]. The concept of user-centered design refers to this attempt. The fundamental principles of such new design approaches are: involvement of target-users in the design process, action-facilitation design, and scenario-based design. Even if accepting the centrality of the user in the design process is becoming a more accepted prerequisite of appropriate person-machine design, its application has often been limited in practice to some particular design stages. A look at the design cycle that Wickens, Gordon, and Liu [7] describe will be sufficient to show this. At the beginning of the cycle, potential users rarely converse with designers. It is the "human factors professionals," sometimes psychologists, sometimes ergonomists, who provide designers with the frame of reference concerning the task, the work environment and users' needs. As the prototype is developed, users are more easily included in the design process, especially for the validation of the prototype. At the end of the design process, the functionality of the product is assessed sometimes in real use, for a period of time. However, at this late stage, changing the product becomes unfeasible and procedures or training measures constitute, for the most part, the protective measures that ensure safety.

Conducted in this way, none of the above stages relate specifically to a user in context centric view. The process places the product at the center.

From an activity-theory perspective, aid systems should be designed to support operators in doing a task safely and efficiently in real work situations. The concept of action facilitation was introduced by Roe [8] and is based on the theoretical work of Hacker [9]. Following Arnold [10], the action facilitation approach generated an approach to the design or redesign of mental information work. Cognitive activity analysis as developed by Rasmussen and Vicente [11], is placed at the center of the analysis, focusing on information, mental effort, decision making, and regulation. The concept of ecological interface was developed to illustrate an interface that provides appropriate support for the different levels of cognitive functioning.

Along the same line, but this time stressing the contextual point of view, is the scenario-based design approach, a set of perspectives linked by a radical vision of user-oriented design [12]. This approach is not entirely new. For decades, systems de- 
velopers have spontaneously used scenarios to envision future concrete use of their systems. However, this informal practice has gained international acknowledgment, and the social content of the work is taken into account. To integrate context into the design, the task analysis stems from a scenario: "One key element in this perspective is the user-interaction scenario, a narrative description of what people do and experience as they try to make use of computer systems and applications. Computer systems and applications can and should be viewed as a transformation of user tasks and their supporting social practices" [12, p 3]. Despite these valuable insights, scenarios constitute only examples of interactions of use and thus suffer from incompleteness. In a recent contribution, Vicente [13] proposes a formative approach to work analysis giving the workers some responsibility to "finish the design" locally as a function of the situated context, thereby taking into account the variability of the world and creating the conditions for productive adaptation.

Hereafter, we use two series of studies to illustrate how important work analysis is in evaluating new technology in order to identify critical dimensions of work and anticipate the impact of the devices on these dimensions, one looking at a new automatic drug device for anesthetists and one at a new robotic surgery system. More than $600 \mathrm{~h}$ of observation were conducted in the operating rooms selected on the basis of their use of the two new devices.

\section{CASE EXAMPLES}

\section{A. Example 1: Anesthesia Automatic Drug-Infusion Device}

The last 20 years have been decisive in anesthesia because of the evolution of the drugs that are used and the technological evolution of the equipment. In anesthesia, the physician monitors the patient's physical status and administers drugs to control the parameters to desired levels based on patient baselines, disease type, and so on. The clinical signs of the patient-skin color, appearance of the pupils, etc.-are no longer the only source of information for the anesthetist during a surgical operation. Sensors on the patient take data relative to the essential physiologic parameters and report them in the form of values, curves, and graphs usually presented today in an integrated manner on screens. One more step has been taken, however, along the road toward more complete automation. Automatic intravenous administration systems (pumps) are becoming common. Planned and programmed in advance depending on the patient's characteristics and those of the operation to be done, they order the administration of drugs in a continuous and regular manner and free the anesthetist from unnecessary handling. However, the latter continues to take over manually in case of a problem. One can consider that this constitutes the first step toward more automatic control in anesthesia and that because of this, it could be the forerunner to more radical changes (associated with full automatic control, such as has been found in other systems, such as in aviation.

In this work context, as computers become gradually more capable, it is evident that the cognitive consequences of the device in terms of monitoring, planning, and intervening must be considered by researchers and designers [14]. We used the

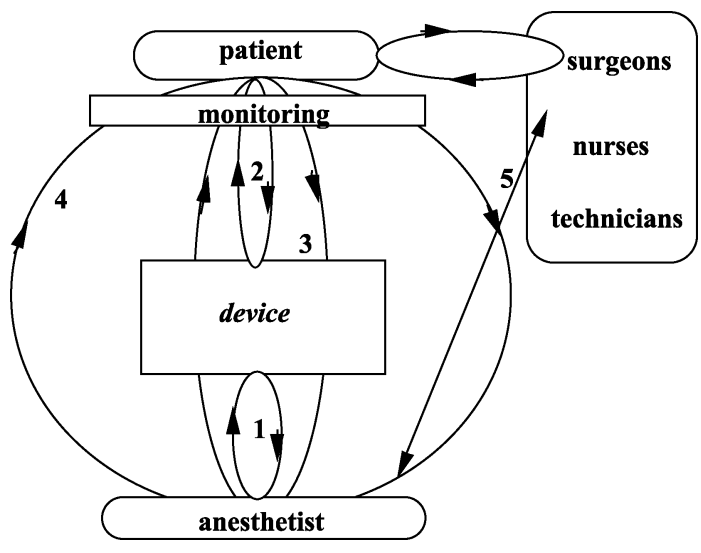

Fig. 1. Loops of control of the H-M system.

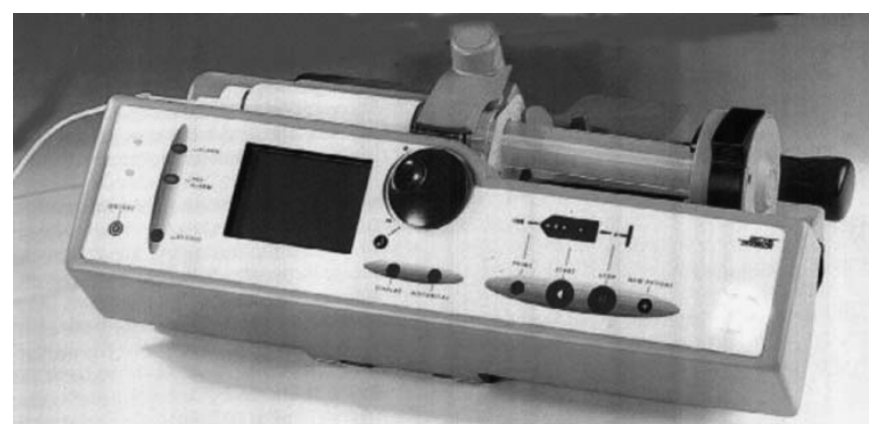

Fig. 2. Anesthesia automatic drug-infusion device.

model of supervisory control developed by Sheridan [15] to illustrate how the device introduces three novel control loops in the human-machine system (Fig. 1).

Without the device, the anesthetist directly injects the drug into the patient and controls the effects through the monitoring screens and the patient (loop 4).

With the device, (Fig. 2) the anesthetist programs data in the system and controls his actions on the device (loop 1). (S)he controls the effects of the actions through the monitoring and the patient (loop 3).

In the case of a fully automatic device (actually in study), the device acts directly on the patient without any human's action (loop 2), directly connected to human's consciousness level.

The impact of the device on social interactions must also be considered (loop 5). In an operating room, the anesthetist does not work alone. (S)he works in a team and the device might have an impact on the work of the other members of the team.

Without reviewing in detail the results of the evaluation [16], our work analysis reveals that:

The device transforms the knowledge and the communication mode (loop 1, loop 5) [1]. A new way of thinking must be developed by the anesthetists. Used to working in terms of dosage $(\mathrm{mg} / \mathrm{kg} / \mathrm{h})$, the doses having been transformed into flow, from now on, the device works in terms of desired plasmatic concentration and the computer linked to the system calculates the circulation flow to reach the target value [17]. This is only valid for the drug (Propofol) used by the device. The anesthetists still communicate and 


\begin{tabular}{|c|c|c|}
\hline Perception of the patient's condition & ++- & $\begin{array}{l}\text { Adjustment to the patient } \\
\text { Tendency to rely on the system }\end{array}$ \\
\hline The plan of action & +++++++ & Avoid re-injection \\
\hline Memorizing the information & +- & \\
\hline Interpretation of the data & ++ & \\
\hline Ability to react & ++++ & $\begin{array}{l}\text { Frees the hands } \\
\text { Rapid adjustment }\end{array}$ \\
\hline
\end{tabular}

Fig. 3. Answers from anesthetists to the question: "Do you feel that using such a system has consequences on ...? Explain what" (13): (n) number of answers with $+=$ yes and $-=$ no.

\begin{tabular}{|l|l|l|}
\hline Increases the work load & ++++++ & $\begin{array}{l}\text { Programming } \\
\text { Arterial line } \\
\text { Increased vigilance on pressures }\end{array}$ \\
\hline Decreases the work load & +++ & Hands-free =>maintenance \\
\hline Increases patient security & +++++++ & Stabilizes the pharmacocinetic model \\
\hline Changes the induction & +++++++ & Softer for the patient \\
\hline Changes the maintenance & ++++ & Easier \\
\hline Changes the re-awakening & +++++++ & Quicker \\
\hline
\end{tabular}

Fig. 4. Answers from anesthetists to the question: "Do you think that using the system. . ? Explain how" (13). (n) number of answers with $+=$ yes and $-=$ no.

work in terms of $\mathrm{mg} / \mathrm{kg} / \mathrm{h}$ for the other drugs. Frequently, we observed difficulties and errors of conversion from one mode of calculation to another. A new protocol has been developed in order to help the anesthetist in the new way of thinking and programming.

1) New planning tasks come up (loop 5): The length of action of the Propofol is short and the postoperation analgesia must be planned at the time of return to consciousness which means a critical moment for the anesthetist's cognitive care. Furthermore, in a previous article [18], we showed that the principal goal of the anesthetist is to manage the human body's tolerance to the surgical act. This basic statement implies that the actions launched by the anesthetist will not only take into account technical constraints (the constraints of the surgery, the constraints of the patient), but also temporal constraints in order to maintain synchronization between the patient state, the surgical act, and the anesthetist's interventions. The simple fact of introducing a new product with a different time lag requires the anesthetist to restructure their acquired strategies of synchronization and leads, at the first stage of implementation of the device, to several errors of synchronization.

2) Attention allowances are changed (loop 3): Propofol has a hypotensive effect that requires closer attention by the anesthetist to the patient's blood pressure.

3) The system requires the use of prefilled syringes equipped with a system of electronic recognition. New and old syringes exist in the operating room with possible mix-up of syringes under time pressure.

4) The device has an impact on the work of the members of the team (loop 5). It is required to be placed close to the patient and, on several occasions, the surgeon has been bothered by the device. More importantly, several surgeons have said that the new protocol of anesthesia has some side effects such as an increase in bleeding, both during the operation and postoperatively (loop 2). As a result, the new protocol for drug administration was readapted.

Compared to a classical push-syringe, the device is, in general, well accepted by the users. It allows the anesthetist to quickly change the level of anesthesia thanks to a wheel that is very easy to use. The anesthetist can increase, but also decrease, the target concentration that is still not possible with manual drug injection. However, the device increases the number of programming tasks (age, weight, identification of the patient's target plasma concentration) precisely at the beginning of the operation, when the number of acts is already high. Furthermore, observations show that, in certain emergency situations, the anesthetist gives up using it. It is less the instrument than the new anesthetic product, the Propofol, which transforms the cognitive activity. In fact, its pharmacocinetic characteristics-it is a blood pressure reducer with a short half-life-demands close control of the patient's blood pressure and planning of other anesthesia for the reawakening, thus giving additional work to the anesthetist and, more importantly, reporting the critical task of awakening at a next locus to a next person.

The duality between the cognitive aspects, which are more restricting, and the clinical objectives, which are better reached, is reflected in the appraisal that the anesthetists themselves gave for the instrument (see Figs. 3 and 4).

\section{B. Example 2: Robotic Surgery System}

Surgery has also known important developments with technological advances. Laparoscopy is certainly one of them. There is little question that laparoscopy represents a definite progress in patient's treatment. However, there are a lot of drawbacks, 


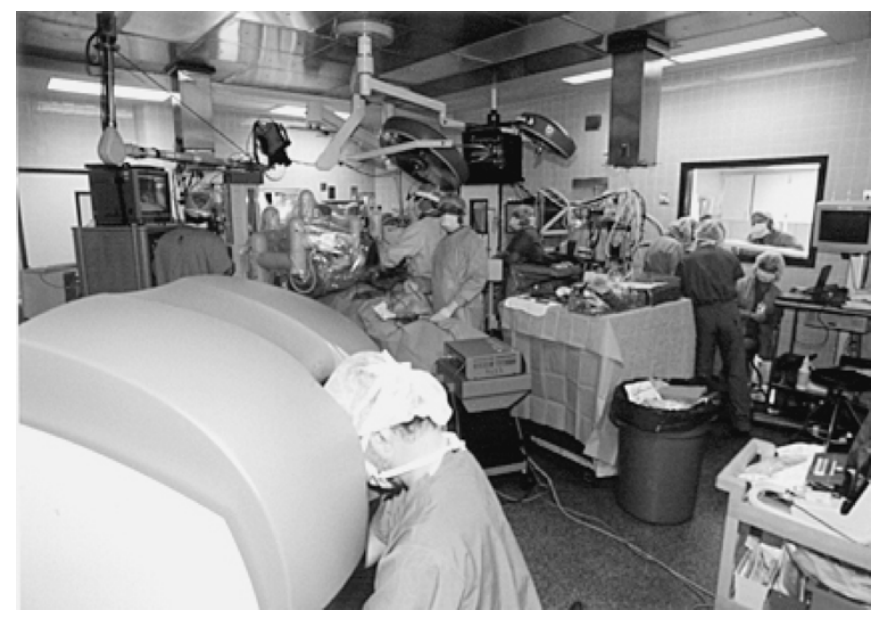

Fig. 5. Operating room with the robotic system.

some of which are not without significance. For instance, the surgeon has lost all tactile feedback, (s)he has to perform operation with only sensory input from the two-dimensional (2-D) picture on a video screen, and the procedure, to be done with long instruments, is seldom performed in a comfortable position for the surgeon. The fact that long instrument are used through an opening (trocar) in the abdominal wall, limits the degrees of freedom of the surgeon to a number of four: in and out, rotation around the axis, up and down and from medial to lateral. The aim of the computer-guided mechanical interface, commonly referred to as a robot, is to allow for 1) restoration of the degrees of freedom that were lost, thanks to an intra-abdominal articulation of the surgical tools; 2) 3-D visualization of the operative field in the same direction as the working direction; 3) modulation of motion amplitude by stabilizing or by downscaling; and 4) remote-control surgery. Because of these improvements, the surgical tasks can be performed with greater accuracy. However, to place a computer as an interface between the surgeon and the patient transforms the system in the same way as it did in aviation.

We performed observations of patients who were submitted to robot-assisted laparoscopic procedures for gastrooesophageal reflux at a Brussel's hospital (Fig. 5) after having obtained a clearance from the ethical committee and after full informed consent from the patients.

Results of work analysis show how the devices introduce some significant changes at the individual and organizational levels concerning:

1) the tasks of each member of the OR team.

2) the collective "situation awareness."

3) the role and status of the members of the OR team.

Concerning the manual tasks, the robot approach implies new operative strategies, including a specific trocar placement in order to accommodate the robot arms. The operative time is comparable with the laparoscopic procedure. However, operative time not only depends on the surgical dissection, but also on the installation of the system as well. This latter step must be performed under the supervision of a technician and takes time.

The use of the robot implies new knowledge not only for the surgeon but for the whole operating team relative to the new pro-

\section{Communication during cholecystectomy}

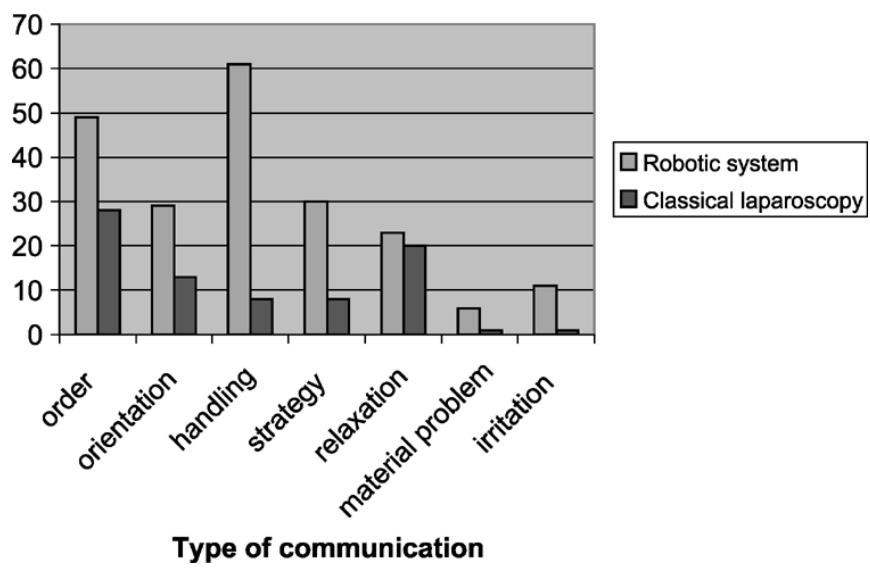

Fig. 6. Comparison of communication between robotic and classical laparoscopy surgeries.

cedure, the placements of the instruments and the preparation of the system.

Although the robot gives a three-dimensional (3-D) image, the optical system is not yet ideal. The 3-D optical system is characterized by a very narrow field of vision. Therefore, surgeon's has continuously to interrupt the dissection for repositioning the lens. These frequent interruptions, as well as the absences of global view of the operative field, might be responsible for the bleeding they encountered while dissecting with the robot system and that made them convert into conventional laparoscopic approach. Better optical systems are needed.

Up to now, tactile feedback are not provided to the surgeon.

Although a 3-D image is provided to the surgeon, the team works with a 2-D picture. A different quality and accuracy of information is provided to the surgeon and the team creating a rupture in the representation of what is going on. We can predict visual orientation will be associated with new forms of errors such as a loss of "collective situational awareness." The changes we observed in the communication exchanges during one robotic and one classical laparoscopy surgeries conducted by the same surgeon with the same team illustrate this evolution (Fig. 6).

Coordination and cooperation between surgeon and surgeon assistants are modified. In classical laparoscopy, the assistant is in front of the surgeon and follows the actions in visu, anticipating some demands of the surgeon. With the robotic system, the fact that the surgeon is at a distal point from the patient requires the use of more frequently verbal communication to coordinate actions (Fig. 6). The surgeon is isolated from the rest of the team and the work becomes more of a solo work. Thus, the allocation of attention of the team is modified. Exchanges between surgeon and assistants concern orders, handling of instruments, strategies, and orientation as shown in Fig. 6.

As a result, the status of the team's members is modified. The surgeon assistant becomes more like a technician, responding to the orders of the surgeon. There are two new actors in the team: the robot and the technician who become essential members within the team.

The arms of the robot imply a new position for the anesthetist. They are very cumbersome, which makes the conversion, in this 
case, quite awkward. As a result, we can predict a delay at a very critical time.

On October 31, 2002, a robot-assisted surgery in Florida lead to the death of a patient after wrong vessels were cut during a kidney operation. One can think that the side effects cited above have played a role in the occurrence of the accident.

\section{TOWARDS A NEW TEChNOLOGy EVAlUation GRID}

Clearly, the samples of studies cited above have shown that problems with new technology in complex systems such as healthcare occur not only because of the characteristics of the device per se but because of the use of the device in the context under time and resource constraints. Context-free evaluations, which are usually conducted in healthcare systems, are unlikely to uncover the important problems of work situation and determine why they are important. This implies the use of different methods of analysis in order to identify criteria that more successful systems should meet. Only work analysis based on observations of user interactions in the field provides this information. One original aspect of our work comes from the fact that it concerns two groups of users (anesthetists and surgeons) in one same context (the operating room). It appears that the critical dimensions and thus the criteria that designers should take into account differ from one viewpoint to the other. In case 1, we extracted monitoring and planning constraints, crucial in the anesthetist's activity and showed how the very act of introducing new products has an impact on these constraints and affect anesthetist's cognition. In case 2, we extracted the organizational structure of the surgeon's work and illustrated the impact of the new device on the physical and social interactions between the actors. The distance introduced by the system influences the communication and changes the role of the members of the team. Since communication provides information, and since information is often perceived as control, it soon becomes clear that the impact of the device on communication will play a large role in how the user and the team perceive, accept and use the device.

Given these remarks, we developed a methodology for the evaluation of new technology in complex systems based on work analysis [16]. Our aim is to insist to assess the impact of technology changes on all the dimensions of a work situation: technical, performance, cognitive, organizational, and economic. Which dimensions and criteria are developed in more details depends on the results of the work analysis. The list proposed below is not exhaustive. For each dimension, we describe:

1) the evaluator (user, expert, designer, etc);

2) the dimension and the criteria measures;

3) the source of data used;

4) the phase of the evaluation.

\section{A. Technical Dimension}

"Evaluation of the technical objectives and verification to what extent they are met in the context"

The evaluation of this dimension is multidimensional. The expert (analyst) can evaluate the compatibility of the use of the system in the context with the technical prerequisites of the device based on the documentation and observation. (S)he can also analyze the reliability of the device in the context. Two kinds of studies can be made: experimental study often realized by the designer in collaboration with the users and field study based on the observations of problem interactions (scenario-based). Even if the results of our studies showed the interest of these systematic observations, they are rarely organized by the designer. Most of the time, feedback returns to the designer whenever a critical problem has occurred.

\section{B. Clinical Dimension}

"Evaluation of the compatibility of the device with the clinical objectives pursued"

Many of information technologies do not have clinical effects even in the health domain (monitoring device, communicative tools, etc.). In this case, this dimension does not have to be considered. However, if the device has clinical effect, the analyst must assess at what extent the clinical objectives are met in the context. This dimension requires the collaboration of the users and the designer.

\section{Cognitive Dimension}

"Evaluation of the impact of the device on the practitioner strategies, analyzed in a cognitive framework"

To evaluate this dimension, the analyst assesses the impact of the device on the information processes for each practitioner. (S)he measures the impact of the device on the activities of information observation, of decision making (including anticipation and planning) and of execution on the basis of observations and questionnaires. Depending on the type of technology, (s)he will develop a detailed analytical evaluation of the functionality of the information presentation within the man-machine interaction. In some cases, observations of problem interaction before, during, and after the phase of implementation are more important. These required an analyst familiar with the work situation and with the device.

\section{Organizational Dimension}

"Evaluation of the impact of the device on the team and work organization"

The development and complexity of technology, along with the intensification of specializations, have contributed to making collective work indispensable. The implementation of new technology changes the team and the work organization. The evaluation of this dimension is multidimensional. Impact can be measured on the role and status of the actors, on the communication and on the cooperation strategies.

\section{E. Economical Dimension}

"Evaluation of the impact of the device in terms of economic effects"

Each use of technology has an economic effect. This effect can influence the usability of the device. Effects measures in this dimension are numerous. Often costs-benefits analyzes are made by economists and focus on direct running costs. Benefits 
derived from cognitive or organizational or clinical effects are more difficult to quantify.

We applied the method in a systematic way to the two devices cited above, one month after the implementation and three months after in order to be able to separate the phenomena linked only to the start-up phase from the others. As a result, a feedback loop was created between designers and users but this was at a stage where protocols and training constituted the only measures possible to improve the usability and the safety of the device. Nevertheless, the feedback loop appears an important factor to motivate people to go on using the devices.

\section{CONCLUDING REMARKS}

Ergonomic criteria are receiving increasing attention from designers but their application doesn't ensure that technology matches the user's abilities, the user's needs and the user's work constraints. Being used in a work situation, the tool changes the way of doing things. Although we cannot predict the future, we may attempt to better guide the design process by taking into account the critical dimensions of work in context under time and resource constraints. Only work analysis using different techniques to collect data (field observations, questionnaires, experimentations) can provide a useful window into these critical dimensions and thereby a systematic basis for designing devices that workers need in context. The method of evaluation developed above is rather a guide than a close method. Its principal aim is to adopt a system viewpoint of work. In healthcare, as in other complex systems, there is a need for researchers and designers to investigate the impact of the devices on the reciprocal interaction between cognition and organization. Doing so is critical for the quality, safety, and effectiveness of the modern world.

\section{REFERENCES}

[1] L. Bainbridge, "The ironies of automation," in New Technology and Human Error, J. Rasmussen, K. Duncan, and J. Leplat, Eds. London, U.K.: Wiley, 1987, pp. 271-283.

[2] R. O'Moore, "The conception of a medical computer system," in Assessment and Evaluation of Information Technologies in Medicine, E. M. S. J. van Gennip and J. L. Talmon, Eds. Amsterdam, The Netherlands: IOS Press, 1985 , pp. $45-49$.

[3] T. B. Sheridan, "Human Centered Automation: Oxymoron or Common Sense?," presented at the Industrial Summer School Human-Centered Automation, Saint-Lary, France, 1995.

[4] D. A. Norman, The Invisible Computer. Cambridge, MA: MIT Press, 1999.

[5] - Things That Make Us Smart: Defending Human Attributes in the Age of the Machine. Reading, MA: Addison-Wesley, 1993.

[6] C. E. Billings, Aviation Automation: The Search of a Human-Centered Approach. Mahwah, NJ: Erlbaum, 1997.

[7] C. D. Wickens, S. E. Gordon, and Y. Liu, An Introduction to Human Factors Engineering. New York: Longman, 1997.
[8] R. A. Roe, "Acting systems-design—an action theoretical approach to the design of man-computer systems," in The Meaning of Work and Technical Options, V. De Keyser, T. Qvale, B. Wilpert, and S. A. Ruiz Quintanilla, Eds. Chichester, U.K.: Wiley, 1988, pp. 179-195.

[9] W. Hacker, "Activity: a fruitful concept in industrial psychology," in Goal Directed Behavior: The Concept of Action in Psychology, M. Frese and J. Sabini, Eds. Mahwah, NJ: Erlbaum, 1985, pp. 262-383.

[10] A. G. Arnold, Action Facilitation and Interface Evaluation. A Work Psychological Approach to the Development of Usable Software. Delft, Holland: Delft Univ. Press, 1999.

[11] J. Rasmussen and K. L. Vicente, "Coping with human errors through system design: implications for ecological interface design," Int. J. Man Mach. Studies, vol. 31, pp. 517-534, 1989.

[12] J. M. Caroll, Scenario-Based Design. Envisioning Work and Technology in System Development. New York: Wiley, 1997.

[13] K. Vicente, Cognitive Work Analysis. Toward Safe, Productive, and Healthy Computer-Based Work. London, U.K.: Erlbaum, 1999.

[14] R. Cook and D. Woods, "Adapting to new technology in the operating room," Human Factors, vol. 38, pp. 593-613, 1996.

[15] T. B. Sheridan, "Supervisory control," in Handbook of Human Factors, G. Salvendy, Ed. New York: Wiley, 1987, pp. 1243-1263.

[16] A. S. Nyssen, V. De Keyser, A. d'Hollander, and M. Lamy, Anesthesistes et patients devant le risque d'erreur: Développement d'une méthodologie d'évaluation des systèmes informatisés de contrôle et de surveillance. Evaluation longitudinale de l'impact des nouveaux systèmes de contrôle et de surveillance [Anesthetists and patients faced with human error: Development of methodology for the evaluation of automated systems for control and monitoring systems. longitudinal evaluation of the impact of new control and monitoring systems], ULG, Belgium, Dec. 1999.

[17] F. Engbers, "Practical use of diprifusor systems," Anaesthesia, vol. 53, p. <<AU: PAGE RANGE AND ISSUE NUMBER?>>, 1998.

[18] A. S. Nyssen and D. Javaux, "Analysis of synchronization constraints and associated errors in collective work environments," Ergonomics, vol. 39, pp. 1249-1264, 1996.

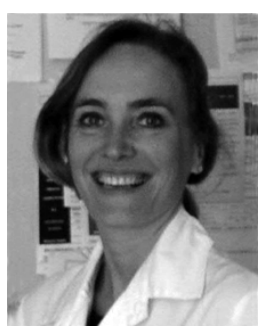

Anne-Sophie Nyssen received the Ph.D. in work psychology from othe University of Liege, Liege, Belgium, in 1997.

She is a Doctor of work psychology and a Professor at the University of Liege, Belgium. Her main interest is human error in cognitive complex systems. She has collected anesthesia-related human errors by interview and direct observations in operating rooms, and has identified the role of contextual, cognitive and organizational variables on the production of these errors. Progressively, the research extended its scope and an evaluation methodology was developed to assess the impact of technology changes on practitioner cognition. For the last ten years, she has contributed to the use of a full-scale anesthesia simulator for training and research on expertise. In 1999, she performed post-doctoral research at Stanford University, which supported by a grant from NATO. In the same year, her research project to develop a system-wide healthcare critical incident reporting system in Belgium was approved for funding by the Office of the Prime Minister of Belgium. Since then, her research domains have been extended to other complex systems such as transportation, steel industry, etc. She has investigated a variety of work dimensions such as the impacts of new technology on the work, cognition, and error; how to make aid systems based on work analysis and expertise extraction; decision-making strategies in crisis situations; time aspects of work situations (representation and cognition); accident analysis. Central to her lab is the use of multiple techniques in order to understand the complexity of work systems: in situ observation, questionnaire, interview, observation of performance in simulated situations, and spartan lab settings. 